\section{Effects of occupational exposure to glyphosate in winegrowers}

\author{
Melissa Ferrian, 1 \\ Antonella Annicchiarico, 2 \\ Mario Olivieri, ${ }^{1}$ Claudio Colosio, ${ }^{3}$ \\ Emanuela Corsini, ${ }^{4}$ \\ Alessandra Barassi, ${ }^{3}$ \\ Giuseppe Mastrangelo, 5 \\ Emanuela Fadda,5 Manuela Peruzzi,6 \\ Stefano Porru1,7 \\ 1Department of Diagnostics and Public \\ Health, Section of Occupational \\ Medicine, University of Verona, Verona; \\ 2Postgraduate School of Occupational \\ Medicine, University of Verona, Verona; \\ 3Department of Health Sciences, \\ University of Milan, Milan; ${ }^{4}$ Department \\ of Environmental Sciences and Politics, \\ University of Milan, Milan; 5 Department \\ of Cardio-Thoraco-Vascular Sciences and \\ Public Health, University of Padua, \\ Padua; 'Unit of Injury Prevention, \\ SPISAL ULSS 9 Scaligera, Verona, Italy; \\ 7University Research Center "Integrated \\ Models for Prevention and Protection in \\ Environmental and Occupational \\ Health" MISTRAL Universities of \\ Brescia, Milano Bicocca and Verona, \\ Italy
}

\section{Abstract}

Glyphosate is a non-selective systemic herbicide used in agriculture. For almost half a century, the International Agency for Research on Cancer has run a Monographs program, the conclusion in March 2015 that glyphosate is "probably carcinogenic to humans" in addition to being genotoxic and carcinogenic in animals, while the regulatory European Food Safety Authority have asserted that glyphosate poses no public risk. The scientific debate is still lively. We collected detailed socio-demographic, occupational exposures and health surveillance information for 26 winegrowers as aim to investigate exposure to glyphosate and other pesticide. Exposure was assessed through biological monitoring (24-hour urine collection), immune function (IL-4, IL-5, IL-8, IL-12, IL17, IL-33, IFN- $\gamma$ ), transcriptional and post transcriptional alterations (miRNA) and genotoxic effects (Comet assay). The exposure conditions in our winegrowers, as referred to the parameters so far analyzed, did not reveal a significant glyphosate absorption nor significant health concerns.

\section{Introduction}

Glyphosate is a widely used herbicide. Within occupational settings, most relevant routes of absorption are via inhalation and skin. Since 2015, the International Agency for Research on Cancer (IARC) classified glyphosate as a probable human carcinogen, referring to haemolymphopoietic system as the main target, while the European Food Safety Authority (EFSA) concluded that it is unlikely that glyphosate poses a carcinogenic hazard to humans, taking into account the CLP classification. 1-3 The scientific debate is still lively. Until now, there are no studies simultaneously evaluating, in winegrowers exposed to glyphosate and other pesticides, indicators of exposure and effect through biological monitoring, immunological indicators, genotoxicity and regulators of gene expression.

Our study aimed to investigate exposure to glyphosate and other pesticides in winegrowers as well as potential effects regarding genotoxicity, immunomodulation and gene expression. The study was carried out within the framework of the Regional Plan for Prevention 2014/18 denominated "Prevenzione degli infortuni e malattie professionali in agricoltura".

\section{Materials and Methods}

By means of questionnaires, we collected detailed socio-demographic, occupational exposures and health surveillance information for 26 winegrowers; quantities, timing and ways of glyphosate and pesticides use were also collected through official health authority registers. As for biological monitoring of exposure, 24-hour urine glyphosate was chosen as the indicator which was evaluated pre and post application. The following analyses were performed before and after the use of glyphosate during the period of pesticides treatments: we monitored general blood chemistry parameters, immune function (IL4, IL-5, IL-8, IL-12, IL-17, IL-33, IFN- $\gamma$ ), possible transcriptional and post-transcriptional alterations (miRNA), potential genotoxic effects (Comet-assay on lymphocytes DNA). Statistical analysis used Wilcoxon signed-rank test and three-way variance analysis with repeated measures after normalizing the outcome variables with appropriate linearizing transformations.

\section{Results}

In our study population, glyphosate exposure was low in quantity, limited in duration and appropriate in mode. Biological monitor-
Correspondence: Stefano Porru, Department of Diagnostics and Public Health, Section of Occupational Medicine, University of Verona, Verona, Italy.

E-mail: stefano.porru@univr.it

Key words: Glyphosate; winegrowers; comet assay; miRNA; immune function.

Conference presentation: this paper was presented at the Second Centro 3R Annual Meeting - 3Rs in Italian Universities, 2019, June 20-21, University of Genoa, Italy.

Received for publication: 28 October 2019 Accepted for publication: 11 November 2019.

This work is licensed under a Creative Commons Attribution NonCommercial 4.0 License (CC BY-NC 4.0).

(C) Copyright: the Author(s), 2019

Licensee PAGEPress, Italy

Biomedical Science and Engineering 2019; 3(s3):110 doi:10.4081/bse.2019.110

ing did not show high absorption rates; immunologic tests seemed to show some modification (after $v s$ before usage), limited to IL-4, IL-5, IL-5, IL-8 and IFN- $\gamma$. Genotoxic alterations were not evident. Further statistical analyses are in progress for the remaining indicators.

\section{Conclusions}

The exposure conditions in our winegrowers, as referred to the parameters so far analyzed, did not reveal a significant glyphosate absorption nor significant health concerns. Potential effects due to the use of glyphosate and other pesticides on immunomodulation, as well as on gene transcription and post-transcriptional regulation are currently under investigation.

\section{References}

1. EFSA. Conclusion on the Peer Review of the Pesticide Risk Assessment of the Active Substance Glyphosate. EFSA J 2015;13:4302.

2. Portier CJ, Armstrong BK, Baguley BC, et al. Differences in the carcinogenic evaluation of glyphosate between the International Agency for Research on Cancer (IARC) and the European Food Safety Authority (EFSA). J Epidemiol Community Health 2016;70:741-5.

3. IARC. IARC Monographs Volume 112: evaluation of five organophosphate insecticides and herbicides. International Agency for Research on Cancer; 2015. pp. 321-412. 